\title{
Web-based Architecture for Mobile Learning
}

\author{
Mohammed Alzaabi ${ }^{1}$, Jawad Berri ${ }^{2}$, Mohamed Jamal Zemerly ${ }^{1}$ \\ ${ }^{1}$ Khalifa University of Science, Technology and Research, UAE \\ ${ }^{2}$ Research and Technology Division, Sonatrach, Algeria
}

\begin{abstract}
The availability of multimedia web resources and the development of mobile phones are two major factors that have opened the way towards the development of M-learning systems. These systems are designed for nomadic learners; they are designed to deliver learning material that adapts to the learner context. In this research we developed a mobile learning system that exploits existing web resources to generate learning material which fits the actual needs of the learner. The system constructs a personalized learning web for a mobile user who needs to learn about a specific topic. The system manages the gathering, authoring, packaging and delivery of learning material. The architecture of the implemented system uses a set of web services to retrieve information and learning resources from the web. These learning resources are packaged into lightweight learning objects designed according to preset layouts. Besides, the system constructs an ontology-like hierarchical content structure to organize learning objects into a learning web allowing the learner to navigate freely according to his/her needs and constraints. The paper presents the design, implementation and an evaluation of the system.
\end{abstract}

\section{Introduction}

Authoring of learning content is a consuming task of courseware engineering. It is a major bottleneck facing widespread use of learning technologies. Educators and researchers have put lot of efforts to overcome this barrier and many systems and tools have been developed to automate various authoring tasks. This has been possible especially with the advent of technologies and standards that helped the creation, packaging, deployment and sharing of learning resources over the web. Furthermore, the emergence of the Semantic Web initiative, which uses descriptions of Web resources, has improved the current state of the Web by enabling knowledge sharing on the Web [1]. E-education can benefit a great deal from available multimedia web resources if those resources are used within a well defined knowledge framework. This will save time and efforts, and authoring will exploit existing resources to the benefit of educators and learners as well. This tendency represents a research direction for many disciplines at the crossroads of knowledge such as Eeducation, Semantic Web and Knowledge Management.

The development of E-education needs to take into account the new technologies for the advantage of learners. As a matter of fact, with the widespread use of mobile phones by a large population, it is possible for users to get access to learning through their mobile handsets while facing real-life situations. Mobile Learning (M-Learning) opens the space-time boundaries of learners; they can learn anytime, anywhere [2]. M-Learning is a stand-alone learning paradigm that has particular educational characteristics, learning environment constraints and supporting technology. Learning via mobiles can be seen as a "first-aid" knowledge acquisition while the learner is facing a real-life situation and is interested to know urgent information about a specific topic of interest. It is a design-to-time learning where the learner is generally constrained by the time he/she affords to spend acquiring knowledge [3]. MLearning meets the learning objectives efficiently if it is context-aware allowing the learner to get finetuned knowledge adapted to the learning environment. This requires a system that is capable of sensing the context and reacting to any change.

Mobile devices offer an attractive environment for learning, however they have inherent technological constraints that need to be taken into account such as the device capabilities (screen resolution and size, memory storage limitation, software constraints, energy consumption), the network bandwidth and the communication cost.

The work presented in this paper is a step forward towards the development of an ecosystem where the available information in the web is utilized in the education field allowing users to learn in context. We present a mobile learning system which makes use of available multimedia web resources to manage the authoring and delivery of learning material for nomadic users. The user interacts with the system by enquiring about a particular topic of interest to fit his curiosity while facing a real-life situation. The system constructs in real time a personalized learning structure that we call a Learning Web (LW) which is mapped from an ontology-like hierarchical structure generated automatically from information gathered in the web. The learning content is packaged into 
preset layouts and presented to the user based on his profile and the capabilities of the mobile handset. The architecture of the system is web-based which is designed as a set of modules that i) gather multimedia information from different locations in the web, ii) package the content into lightweight Learning Objects (LOs), iii) organize LOs into a learning web structure, and finally, iv) deliver the whole to the user allowing him/her to navigate through the learning web according to his/her needs and constraints.

This paper is organized as follows: Section 2 presents related work focusing on similar systems developed in the field. The third section is dedicated to the design of the system. Section 4 shows how our system makes use of structured information in the web to organize the learning sequence of LOs. Section 5 presents the system architecture. Section 6 is dedicated to a case study. Section 7 offers an evaluation of the system. Finally, we conclude this work and propose future research directions.

\section{Related work}

There are many real-life applications that have been developed in the mobile learning field. In [4], the authors presented an adaptive m-learning system. They emphasize the importance of delivering adaptive learning contents according to two perspectives. The first perspective is the learner abilities, which has been constructed according to Bayesian inference mechanism that facilitates the diagnostic procedure of the abilities of learners to build a full learners model. The second perspective is the learning preference by the specification of the mobile device used. It has been implemented using content adaptation technique that transforms the learning content to an adaptive content with the device specification taken into account. By using Bayesian inference mechanism, the authors succeeded to monitor the learning behavior of the learners and assembled a learning style based on the information concluded from learner's activities such as the average time of reading a webpage, frequency of accessing web forums, attending videoconferences and reading materials. With content adaptation technique the authors used a splitting approach for text content adaptation to split the large text into series of smaller texts so that they are properly displayed on the device screen. In [5], the authors proposed a Flash-Based mobile learning system for learning English as a second language (FML4ESL). The system is developed using Adobe Flash CS3 that works with mobile phones which support Adobe Flash lite. The system is designed with the following major objectives: the system provides full control by the users over the mobile device and the lessons; users can listen to the device's speakers reading a text or lesson; users can read the text on the screen while listening; users also can learn conversation rules, reading rules, grammar and spelling. In addition to that, there are exercises after each unit that tests the users about what they have learned. In an early initial work [2] the authors proposed an M-Learning system that is context aware and which takes into consideration the adaptivity of learning and the context of the environment. The system uses domain ontology as a backbone to facilitate the delivery of learning material according to the context and some specific constraints. The system can provide a personalized and adaptive learning experience that takes into account the specification of the mobile device used, learner preferences, speed of learning and background experience. The authors proposed a set of ontological rules that are used in context acquisition to deliver suitable knowledge the learner needs using his device in an allotted time-frame. The architecture of the system is divided into three main layers namely: the context layer, semantic layer and resource repository layer. The context layer handles learner interaction, used device and network connectivity. The semantic layer includes the context acquisition and management component. The latter is a reasoning module that aims to derive the right Learning Web for a given context. The third layer is the resources repository layer which includes all the repositories used by the system. WiCom is another similar mobile application that offers dynamic multimedia content retrieval in distributed environment aimed at journalist [6]. WiCoM enables a reporter to capture events in real-time using their mobile phones and update their server on the latest events. WiCoM uses J2ME on the client side and PHP on the server side and the communication between the client and server is established using GPRS. The reporter can upload, edit, and remove textual and multimedia contents in the server. Another mobile user-centered image and video retrieval application is described in [7]. The application offers acquisition, enhancement, storage, retrieval and delivery of mobile multimedia to the user. Another mobile information retrieval system that discusses the profiling of user actions to extract content based and location based user profiles is described in [8]. The system offers information filtering aimed at matching dynamically the user profile. The system constantly searches for information and documents that are of interest to the user and pushing this information to his/her mobile. This idea is similar to mobile advertising.

\section{System Design}

The system is designed to promote a learning approach that is flexible, adaptive and user-friendly. It is flexible since a learner is not bound to view a predetermined set of learning objects; it is the only 
choice of the learner to be exposed to any learning object he/she is interested in to satisfy his curiosity. The system is adaptive to the needs of the learner; the material proposed to the learner in all learning objects is meant to fit the learner's profile and interests. This is done through a set of layouts that embed variable multimedia objects gathered from the web. Accordingly, a learning object may contain for instance more text, more images or more videos to be proposed to the learner depending on the learner profile and his preferences in the way he/she wish to interact with the system. In our design of the graphical user interface (GUI), we took care of designing a system that is user-friendly to be used by a variety of users. Hence the text to be proposed should not occupy the whole screen of the mobile handset. Moreover, the user should be able to view a learning object in the screen along with hyperlinks to other LOs and control keys with no (or minimum) scroll down. Hence, the GUI design trades-off gracefully the information presented and the ease of use.

\subsection{Learning Content Gathering}

When the user enquires about a certain topic his query is sent to the web for information gathering. The gathered information is processed, filtered and organized into a learning web. The learning web is displayed to the learner from general information to more detailed.

The existence of this feature provides an interesting functionality to the system especially while combined with LO templates. Using templates allow the packaging of the content to be dissociated from its gathering. The main benefit is that different learners will be exposed to the same content presented in different templates to suit a variety of users.

\subsection{System adaptivity}

One of the challenges associated with the use of mobile devices for learning is the size of the screen. Content management must be carefully considered to be suitable for different types of devices. To facilitate this type of management, the delivery of learning material is adaptive to better suit the learner and the mobile device used. Adaptivity can be performed in multiple ways and for this purpose various techniques can be used to get all needed data. In the proposed system the profile of the user is retrieved and the mobile device specifications are sensed at each new session. The former one is essential since it can provide personal information about the user. This information such as the gender and the age of the learner are used to help in constructing a layout that is appropriate for a certain type of users. For example, teenagers prefer to get more images and videos than reading long texts; however adults prefer to focus on getting a basic idea from reading before having images or videos. The specification of the mobile device used is also gathered and accordingly, the contents are displayed on the user's mobile screen. For instance, the size of a picture will be chosen according to the dimensions of the mobile device's screen. This will ensure that the information delivered to the learner is properly displayed. The above gathered information: the learner profile and the mobile specifications are key elements for content adaptation and delivery and offer the learner a personalized experience of learning [9].

\subsection{Content caching}

Once a learner searches for a topic, the server gathers and extracts all information from the web and stores it into the database. In case a learner terminates a session or decides to search for another topic, the information that is already in the database is transferred to a caching component for future fast access. The objective of caching some previously gathered information is to decrease the complexity of the database and to allow a fast access for storage and retrieval.

\section{Ontology-based m-learning design}

Ontology-based learning design is an important ingredient of next generation learning systems. Ontology is defined in [10] as "a body of formally represented knowledge which is based on conceptualization: the objects, concepts, and other entities that are presumed to exist in some area of interest and the relationships that hold them". Ontology is a controlled, hierarchical vocabulary for describing a knowledge system. It abstracts the essence of concepts, allows distinguishing various kinds of objects and defining the relationships among them.

The use of ontologies in m-learning empowers the authoring and delivery of learning systems to provide a standard-based, formal specification with the following benefits [11]: i) standardize m-learning system by reusing and exchanging LOs for a specific field of knowledge. Hence LOs become independent entities of knowledge that can be plugged according to their specification, into any learning web in order to cover the ontology concept fulfillments; ii) efficient LO search by using keywords which are part of the ontology vocabulary in order to provide better context-based search results; iii) adapt learning webs to learners by allowing a great flexibility in the learning object granularity. Thus, an ontology guided LO development will enable production of LOs at different levels of the ontology hierarchy which results in helping authors finding learning resources 
that suit most course requirements; iv) LO sharability and usability allowing authors and learners alike to develop, reuse, update, and maintain LOs at different ontology levels.

Building domain ontologies is not a straightforward task. It is a bottleneck towards developing a robust semantic web capable of filtering appropriate knowledge from the huge unorganized worldwide web. This task needs lots of coordination efforts between domain experts in order to be specified into a formal language and expressed into web markup languages so that to be used by different web communities. Moreover, for applications dealing with various domains, a comprehensive ontology is required for every domain.

In the system we developed we make use of structured information extracted from the web to get around with the inevitable problem of building ontologies to organize the learning web. The idea is to use the Content Section of the famous web encyclopedia Wikipedia (wikipedia.org). The Content Section is a conceptual structure that organizes the information of a document. It is not a comprehensive ontology but for practical applications it can be used as a hierarchical structure to represent the content of a web page into a tree-like structure offering the learner of our system an indepth learning experience from the general topic to the most specific. We chose Wikipedia as our main source of information because it is the most comprehensive source of knowledge available on the web (specifically for the English language), it is written by communities of users and it is growing very fast.

\section{System architecture}

The architecture of the system is presented in Fig.1; it consists of four main modules namely: Web Services Management, Learning Resources Management, Courseware Authoring, and the Graphical User Interface (GUI). The architecture is based on the Client-Server model. At the client side we have the GUI installed on the mobile device which supports either a GPRS or WiFi connection. The main processing is located at the server side. The learning material is fetched on-line form the web using a set of web services; it is packaged and structured and then delivered to the learner. The user equipped with a handheld device interacts with the system by sending a query which includes free keywords representing a specific topic he/she is interested to learn about. The system delivers a Learning Web where the nodes are Learning Objects representing concepts and sub-concepts related to the topic of interest. The learner navigates through the LW by requesting the LOs he/she is interested to be exposed to. In other words, the system guides the learner by indicating the typical flow of learning through the LW but allows the learner to choose the learning path he/she wishes to achieve. In the following sections we present the four modules of our system.

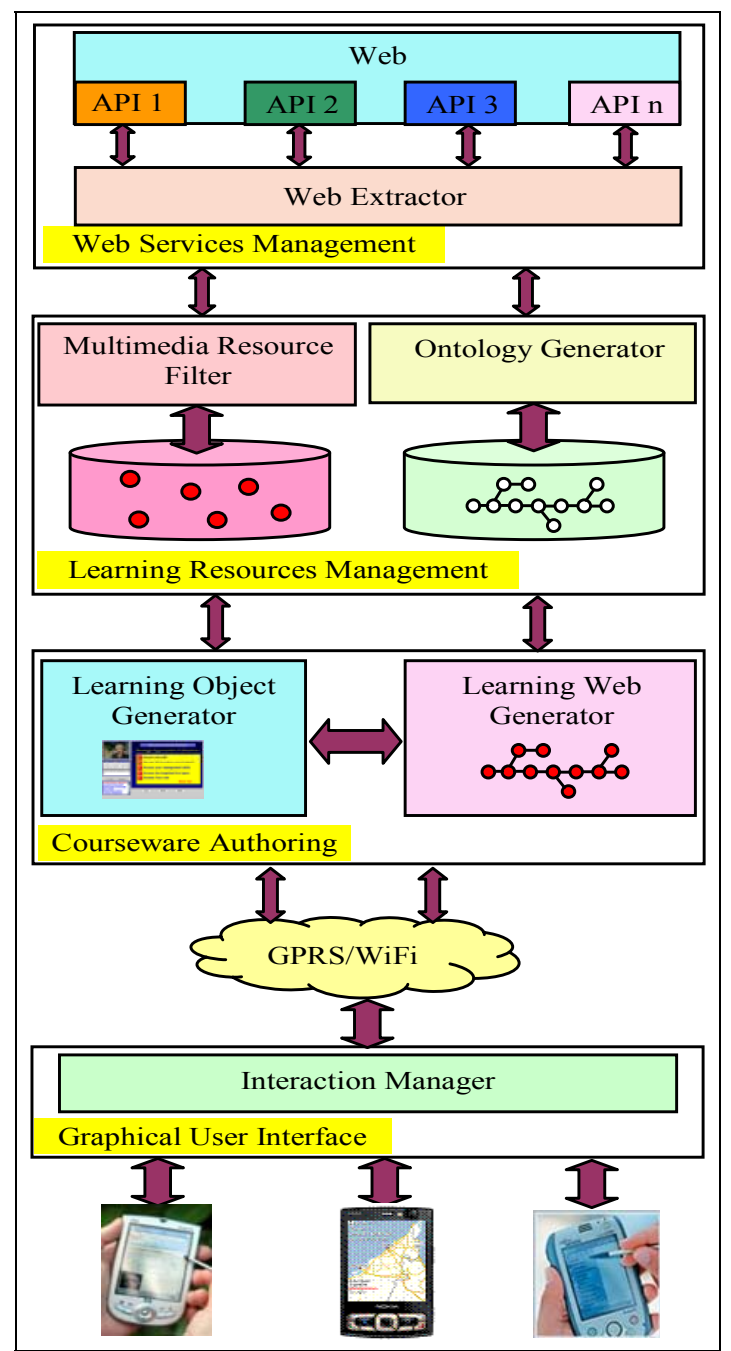

Figure 1. System Architecture

\subsection{Web Services Management}

Web Services Management module (WSM) is responsible of gathering learning material from the web in response to a user query. WSM includes the Web Extractor which invokes different available web services to retrieve multimedia learning resources. Web Extractor embeds a set of programs that have been implemented to request open source APIs (Application Program Interface) in order to use the available web services. Learning resources retrieved from the web consist of text, pictures, and videos. APIs are not standard; each API is developed by a web service provider and made openly available to access its multimedia repository. Hence, in order to access multiple repositories we need to implement a specific program that invokes resources using the 
API of each service provider. This is why we restricted Web Extractor, in a first phase, to deal with specific multimedia web repositories. We used three web repositories in our system i) Wikipedia for the ontology construction and the text content, ii) Yahoo images to retrieve images related to a topic, and iii) Youtube, the video repository to stream videos in our learning objects (Fig. 2).

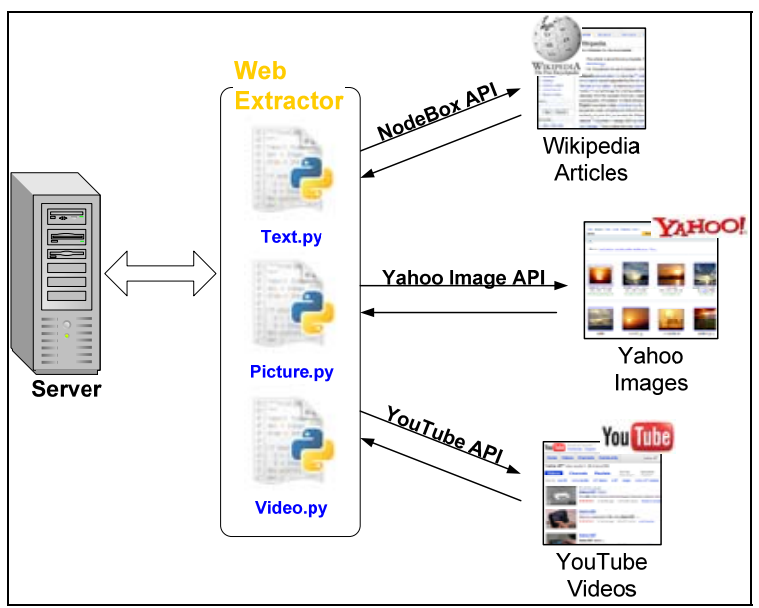

Figure 2. Web Extractor

Wikipedia is one of the resources used in the proposed system. Wikipedia (Wikipedia.org) is a web-based encyclopedia that is considered as one of the essential web resources nowadays. It is an information resource for more than 600 million visitors each year. Moreover, it holds more than 2.7 million articles with 253 languages. As a result, the ability of searching and retrieving information from Wikipedia adds a significant importance to the mlearning application in addition to information trustworthiness. Parsing Wikipedia articles was used in order to get the required information that helps in generating the learning objects.

Yahoo Images (yahoo.com) is the service used in order to support the system with the needed images. Yahoo Image Search is a web service published by Yahoo to let users search the images in the web. It provides a large number of images with different sizes and formats. The API provided with this service has been used in the proposed system to gather variety of images from the web. It also allows the developer to get some details about the retrieved image such as title, URL, small description about the image, width, and height of the image.

The third type of multimedia resources is videos. Google API is used to search YouTube repository (youtube.com) and to retrieve a set of videos that are related to the searched keywords. Furthermore, a set of information about the retrieved videos is found like the format of the video, duration, description, and the location where the video is submitted. Using YouTube API allows the developer to choose the format of the video that is appropriate for his/her environment. It also provides special queries for mobile devices that take the size and duration into consideration. This feature is important for $\mathrm{m}$ learning systems since the time for downloading or streaming the video is of great importance.

Web Extractor has been implemented using Python [12] that is an interactive and interpreted programming language. Python is a scripting language having dynamic type checking. It is used to develop large applications especially for the web since it embeds powerful features for parsing the web pages.

\subsection{Learning Resources Management}

Learning Resources Management (LRM) is the module responsible of filtering, storing and structuring the learning material retrieved by the Web Extractor. LRM consists of two main components namely: the Multimedia Resource Filter (MRF) and the Ontology Generator (OG).

Multimedia Resource Filter ranks the multimedia resources (images and videos) retrieved according to the most relevant to the user's query and profile. MRF uses a ranking function that is currently very basic; that is to select the first one retrieved by the Web Extractor as it is provided by the service provider. However, this function can be refined by considering the service provider's relevance factors such as: most recent, most viewed, best rated and most commented. This functionality is currently under consideration to be added to our system. The learner will be asked upon registration to specify which ranking function he/she prefers and the MRF will retrieve the resources accordingly. The ranking function can be very sophisticated including fine tuned processing techniques from natural language processing which analyzes semantically users' comments added to public multimedia resources. This function will be of great benefit if it is contextaware taking into account the learner's environment. This is the direction we would like to investigate in the future.

All multimedia learning resources are stored into a database in order to allow fast access while deploying the learning object. Moreover, later modules will interact only with the database without the need of web access. The content of the database is stored temporary for the current search session only. Further improvement will consider a session manager which will keep trace of learner sessions.

Ontology Generator is a module that creates an ontology for every session initiated by a user query. As explained in Section 4, it is generated exclusively from the Content Section of Wikipedia. The Web Extractor queries Wikipedia and extracts, using a specific API, the Content Section and also the text related to every title and subtitle of the web page. This content is stored in the database and will be 
retrieved upon learner request. The Content Section is then parsed and processed to produce the hierarchical structure corresponding to the webpage's physical structure that we assimilate to the ontology. Notice that Web Extractor uses mainly title numbering and indentation to generate the physical structure. Once the ontology is generated, it is stored into a database to be used further while creating the Learning Web. Figure 3 shows an example of a Content Section from Wikipedia related to the topic "Python" and its corresponding generated ontology.

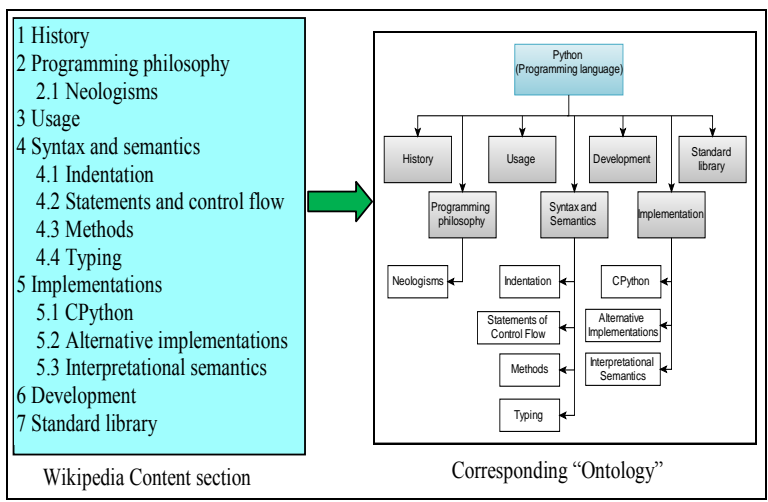

Figure 3. Ontology generation from Wikipedia's Content Section

\subsection{Courseware Authoring}

Courseware Authoring module (CA) includes two components: the Learning Object Generator (LOG) and the Learning Web Generator (LWG). CA is responsible for packaging LOs to be delivered to the user and creating the LW defining the learning course and guiding learner's navigation.

Learning Object Generator gathers all text and multimedia resources from the database and generates a learning object according to the user profile and the specifications of the mobile device used. We have defined four LO layouts that are used by the LO Generator to package LOs. Layouts ensure that each learner category get LOs that suit its specific learning preferences. Users have been categorized by their age into four categories, each one having a specific layout. For instance the layout of the category "teenagers" includes a small introduction text, an image, a hyperlink to a video streaming and hyperlinks to LOs related to the current concept (Fig.5).

Learning Web Generator maps the LW from the ontology. While an ontology is a tree of concepts, the LW is a graph of LOs where each LO is a materialization of a concept in the ontology [9]. The LW guides the learner, by proposing inside each current LO displayed on the mobile handset, hyperlinks to the possible next and previous LOs that he/she can access. This way, the user progression in the courseware is guided during the LW navigation.
Notice that the user is free to choose any route in the LW.

\subsection{Graphical User Interface}

The GUI installed on the user's handheld device includes the Interaction Manager (IM) which listens to user's initiated requests before or during a learning session and invokes accordingly the right service. Additionally, IM manages user's identification by allowing access to registered users who have a stored profile. This functionality allows the system to fine-tune the delivered learning material that is adapted to every user.

\section{Case study}

A major feature or m-learning is to remove the boundaries of time and location associated with learning in order to offer just enough, just in time, and just for me learning experience [13]. To achieve this objective, the proposed system offers the user the possibility to query and search any topic. The learner can get multimedia resources from the web including text, images and videos. In this section we will illustrate the use of our system by showing a test scenario where the user aged 42 years, would like to have knowledge about the topic "python programming language". Further he is interested more specifically in the syntax of the language.

The first step is to log-in into the system; the user types his username and password. Once logged in, the system retrieves all information about the learner from his/her profile in the database. It also identifies the capabilities of the mobile device used by the learner. After identifying all constraints associated with the learner and the device, this information is stored in the database and is valid only for the current session.

The next step, the user types the query "python programming language". The Interaction Manager identifies the keywords from the learner query and sends them to the Web Extractor to start information gathering from the web. All gathered data is stored in the databases including text related to the searched topic, information about the images (URL, title, format, size) and also information about the retrieved videos (URL, title, format, duration). While storing the retrieved article from the web to the database, the system creates the learning web structure including all the entries of the Content Section related to the searched topic and identifies the relationship between them. The system keeps the hierarchy structure by maintaining the "is-a" relation between main titles and the subtitles associated with each main title. The structure generated by the system is shown in Figure 4. The shaded rectangles are the main titles while the white rectangles are the subtitles. 


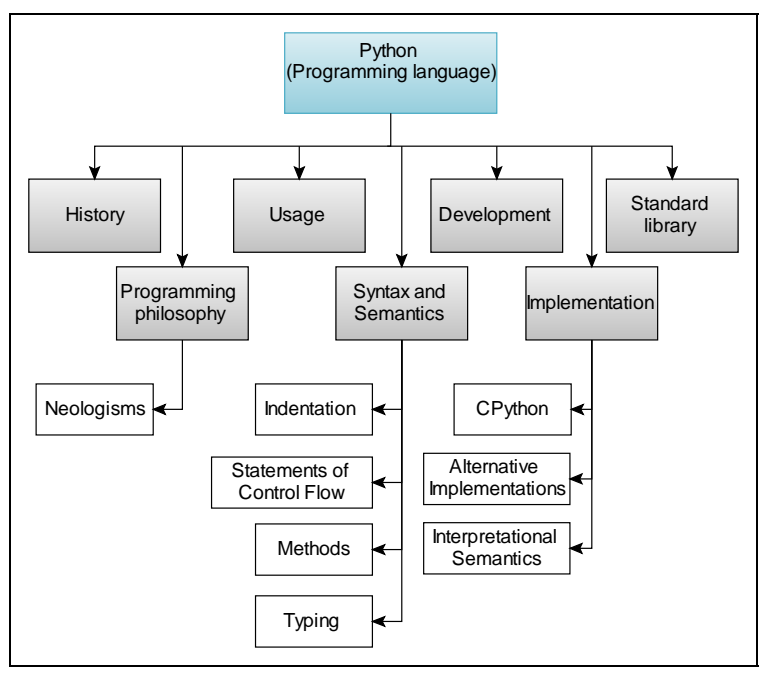

Figure 4. Python Ontology

After generating the content structure based on the information gathered from the web, the system attempts to match the previously stored information of the constraints that are related to the learner and the device used with the layouts provided with the system. The system chooses the best layout according to the learner profile and delivers the multimedia web resources according to the capabilities of the mobile device used. The first LO delivered in the learning web, corresponding to the first title, contains a brief idea about the searched topic (i.e. about python programming language) and two hyperlinks to an image and a video. This layout corresponds to a user aged more than 40 years. Moreover, the system provides the main concepts that are associated with the topic which are represented under "other main titles" label. Figure 5 shows the first learning object delivered by the system.

Python (programming language)
Python is a general-purpose high-level
programming language. Its design philosophy
emphasizes code readability. Pythons core
syntax and semantics are minimalistic, while
the standard library is large and
comprehensive. Its use of indentation as
block delimiters is unusual among popular
programming languages.
read more See image
$\quad$ See video
Other sub titles:
Other main titles:
History
Programming philosophy
Usage
Syntax and semantics
Implementations
Development
Standard library
Influences on other languages

Figure 5. 'Python' Learning Object

The learner would like to investigate more about the syntax of python programming language, then he/she selects the subtopic "Syntax and Semantics" under the "other main titles" topic. The system queries the database about the "Syntax and Semantics" and generates another learning object with the same layout and then delivers it to the learner. The multimedia web resources will also be provided to the learner; however, more concepts are derived from the main topic and will be shown under "more sub titles" label. The topic hierarchy that is related to the above main topic "Syntax and Semantics" is shown in Figure 6.

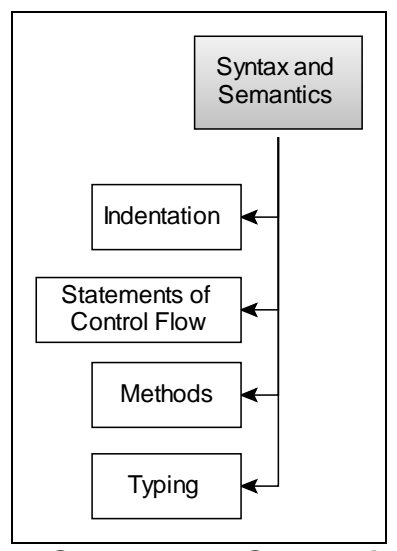

Figure 6. 'Syntax and Semantics' Sub-
ontology

Figure 7 shows the learning object that is specific to the "Syntax and Semantics" topic.

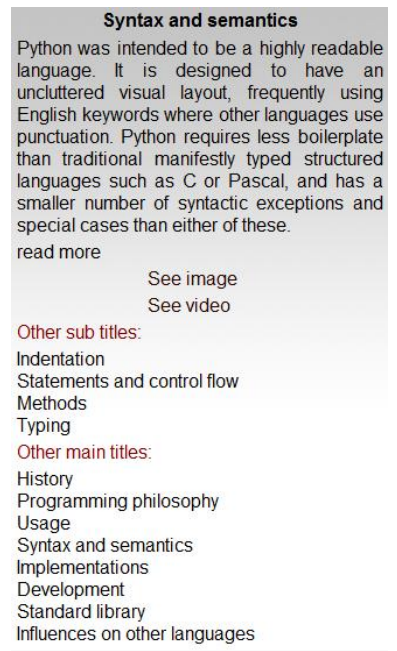

\section{Figure 7. 'Syntax and semantics' Learning Object}

In case the learner decides to see an image or a video, the system renders them in order to fit the device screen. Figure 8 shows some snapshots for the system with different situations.

It is essential to highlight the ability of the system to cache all extracted data from the web and store them in a caching repository. This step increases the efficiency and the functionality of the system 
particularly in case where the learner wanted to search about the same keyword in future sessions.

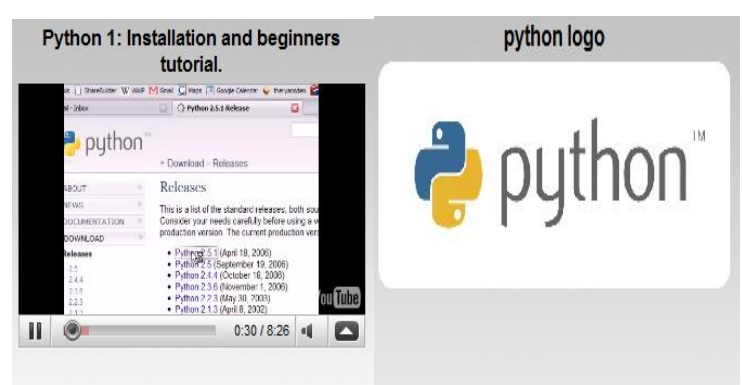

Figure 8. System Snapshots

\section{System Evaluation}

An effective evaluation was conducted with our system. Since the system was mainly designed so that normal and average people can use it without any pre-training courses, people with different educational level were chosen to carry out this evaluation. Three primary factors were used in conducting the evaluation test which mostly oriented to examine the overall functionality of the system, they are: ease of use, flexibility and access to information.

Ease of use factor has a direct impact on the functionality of the system. It can be measured by the time spent by a user in order to learn how to use the system. The less time spent is a good indication for ease of use. Most of the evaluators showed a smooth and fast learning process of how the system is working and what should be done in order to get the desired information about the searched topic. Since the user is only required to enter a keyword after the $\log$ in, the evaluators appreciated the simple steps required for a fully learning experience.

Flexibility is an essential factor that relates the system to more freedom for users. The evaluators valued the ability to specify the layout that can suitably represent their data. Moreover, they liked the way in which the images and videos were rendered in order to fit the screen of the mobile device used. One of the evaluators suggested having a custom layout where users can create their individual layout based on their references.

Lastly is access of information. Most of the evaluators experienced the system in getting some basic information about the searched topic and viewing a picture and a video. However, some evaluators were pleased about the opportunity to have an in-depth learning experience. In addition, some suggestions were proposed to display all data (i.e. text, picture and video) in one screen without any needs to click further links.

\subsection{Cost evaluation}

One of the system features is the ability to connect via Wi-Fi or GPRS for internet connection. In this test, the cost of using both connections was examined and the result is shown in Table 1. Wi-Fi connection can significantly reduce the cost since most of the time it is free of charge. Using GPRS connection, the average downloaded data is found and the associated cost (assuming 1 Fils/KB, 1 \$US $=370$ Fils) is calculated. These results are an average of 5 readings obtained. It was noticeable through the testing that the downloaded data from requesting an image was varying with large difference depending on the size of the downloaded image. Moreover, the data downloaded when requesting a video does not include the streaming data of the video, however it is just the data required to post the video.

\subsection{Performance evaluation}

With this type of evaluation, the time required to deliver the first Learning Web to the learner was calculated. The time was calculated from logging into the system, searching the Web, extracting multimedia information, storing it in the database until creation of the first Learning Web and choosing a layout to be delivered. The layout used in this test was the first layout which contained text, an image, and a video. Table 2 shows all results obtained from the test in more details. It is worth mentioning that the evaluators showed their full satisfaction of the response time when Wi-Fi connection has been used. However, when GPRS connection was used, an acceptable response time was experienced.

Table 1. Data downloaded with the corresponding cost

\begin{tabular}{|c|c|c|c|}
\hline Deliveries & $\begin{array}{l}\text { Average data } \\
\text { downloaded } \\
\text { (Kbyte) }\end{array}$ & $\begin{array}{l}\text { Cost } \\
\text { (GPRS) } \\
\text { \$US }\end{array}$ & $\begin{array}{l}\text { Cost } \\
\text { (Wi-Fi) }\end{array}$ \\
\hline Logging-in & 1 & 0.003 & \multirow{5}{*}{$\begin{array}{l}\text { Free } \\
\text { of } \\
\text { Charge }\end{array}$} \\
\hline $\begin{array}{l}\text { First } \\
\text { Learning } \\
\text { Web (text) }\end{array}$ & 3 & 0.009 & \\
\hline $\begin{array}{l}\text { Request an } \\
\text { image }\end{array}$ & 26 & 0.078 & \\
\hline $\begin{array}{ll}\text { Request } & \text { a } \\
\text { video } & \end{array}$ & 7 & 0.021 & \\
\hline Total & 37 & 0.101 & \\
\hline
\end{tabular}

Another test was done in case the searched topic was already cached in the system. Which means that all extracted data from the Web was available in the cache repository and there was no need for searching the Web again. Table 3 explains the result achieved. 
Table 2. Performance results

\begin{tabular}{|l|l|}
\hline Deliveries & $\begin{array}{l}\text { Average time in } \\
\text { seconds (five readings) }\end{array}$ \\
\hline Logging-in & $1 \mathrm{sec}$ \\
\hline $\begin{array}{l}\text { Search, extract from } \\
\text { Web, store in database, } \\
\text { and create first Learning } \\
\text { Web (text) and layout }\end{array}$ & $6 \mathrm{sec}$ \\
\hline Request an image & $4.25 \mathrm{sec}$ \\
\hline Request a video & $4.5 \mathrm{sec}$ \\
\hline Total & $\mathbf{1 5 . 7 5 ~ s e c}$ \\
\hline
\end{tabular}

Table 3. Performance results after caching

\begin{tabular}{|l|l|}
\hline Deliveries & $\begin{array}{l}\text { Average time (based } \\
\text { on five readings) }\end{array}$ \\
\hline Logging-in & $1 \mathrm{sec}$ \\
\hline $\begin{array}{l}\text { Extract from cache, store } \\
\text { in database, and create } \\
\text { first Learning Web (text) } \\
\text { and layout }\end{array}$ & $3 \mathrm{sec}$ \\
\hline Request an image & $2 \mathrm{sec}$ \\
\hline Request a video & $4 \mathrm{sec}$ \\
\hline Total & $10 \mathrm{sec}$ \\
\hline
\end{tabular}

\subsection{Evaluation against other systems}

One of the criteria used to evaluate a system is to compare it with other similar systems and try to identify main differences in order to enhance the system. Indywiki [14] is one of the software that is quite similar to the developed system. The aim of Indywiki is to visualize Wikipedia browsing. It uses images beside Wikipedia texts in order to provide better way of browsing Wikipedia. It does not only use images related to the search keyword, but also images for links associated with the search keyword. For example, if the user searches for a city in Wikipedia, he/she will get images displayed on Wikipedia page as well as some other images related to other cities in the same country.

To compare between Indywiki and the developed system (i.e. m-Learning system), Indywiki is a Personal Computer (PC) application, while the mLearning system is a mobile application. The later provides more portability, since it can be used with mobile phones which are easy to carry. Moreover, the m-Learning system offers more efficient learning environment to the user. It removes all boundaries of time and place associated with the learning procedure which is not supported by Indywiki. Another comparison is video support. The developed system provides videos related to the search topic, however, Indywiki does not. This feature adds more advantage to the $\mathrm{m}$-Learning system since videos are considered as good source of knowledge for some users.

One of the good features offered by Indywiki is the ability to provide other links to some topics related to the searched topic. It provides more options to the user in case he/she would like to get very detailed knowledge. Both systems use Wikipedia's table of content to provide well structure way of representing the data.

\section{Conclusion}

This paper describes an m-learning system that generates multimedia structured learning content from the web, packages it into lightweight learning objects and delivers it on the learner's mobile handset. We showed in this work that it is possible to make use of existing web resources in learning systems without any prior pre-set knowledge organization and learning object repositories. The system uses a content structure gathered from the web and maps it into a learning web which incorporates the learning objects. The learning web allows learners to get personalized in-depth knowledge about specific topics of interest. The system has the ability to adapt to various constraints associated to the learner profile and the mobile device specifications. A performance evaluation of the system in terms of effectiveness cost of data transferred and response time was provided. A comparison with another similar PC-based system (Indywiki) in terms of features offered was also provided.

The experience is very promising and opens the way to many research directions that need to be explored further. Future work will investigate a webservices architecture that considers various multimedia web repositories from where more consistent content is gathered and delivered to the user. Adapting the content to the learner context in a more systematic way by using a context model (such as location, time, age, interest, etc,) will add value to the system by delivering the right content to the right learner in the right context. Also we would like to consider other languages. Adding new languages will not require a major change in the system architecture but specific APIs need to be developed to deal with some languages. Modeling the user behavior and suggesting similar topics to visit as well as having a proactive system (rather than reactive) based on the profile of the user and past usage and selections are some of the many ideas that make the m-learning system a solid competitor to other types of learning.

\section{References}

[1] Knight, C., Gašević, D., and Richards, G., "An Ontology-Based Framework for Bridging Learning Design and Learning Content". Educational Technology \& Society, 9, (1), 2006, pp. 23-37

[2] E.I. Basaeed, J. Berri, J. Zemerly, and R. Benlamri "Learner-Centric Context-Aware Mobile Learning", IEEE 
Multidisciplinary Engineering Education Magazine, Vol. 2, No.2, June 2007.

[3] E. I Basaeed, J. Berri, J. Zemerly, and R. Benlamri, "Web-based Context-Aware m-Learning Architecture", International Journal of Interactive Mobile Technologies, Vol. 1, No. 1, 2007.

[4] Ho-Chuan Huang, and Fu-Ming Hsieh, "An Adaptive Mobile Learning System with the Support of Learning Diagnosis", Proc. of 16th International Conference on Computers in Education (ICCE), Taipei, Taiwan, October 2008, pp. 189-190.

[5] F. B. Anaraki, "A Flash-Based Mobile Learning System for English as a Second Language", ABAC Journal, September 2008, pp. 25-35.

[6] R. Sivaraman, R. Prabakaran, S. Sujatha, "Dynamic Multimedia Content Retrieval System in Distributed Environment", International Journal of Computer Science and Information Security, Vol. 4, No. 1\&2, 2009.

[7] S. Boll, "Image and Video Retrieval from a UserCentered Mobile Multimedia Perspective", The 4th International Conference on Image and Video Retrieval (CIVR2005), 20-22 July, Singapore, Lectures Notes in Computer Science, Volume 3568, 2005, pp. 18-27.

[8] D. L. Lee, "To Find or To be Found, That is the Question in Mobile Information Retrieval", Proc. SIGIR of Mobile Information Retrieval Workshop, 24 July, Singapore, 2008, pp. 7-10.

[9] Y. Atif, R. Benlamri, and J. Berri, "Learning Objects Based Framework for Self-Adaptive Learning", Education and Information Technologies, Vol.8, No.4, 2003, pp. 345368 .

[10] M. R. Genesereth, and N. J. Nilsson, Logical Foundations of Artificial Intelligence. Morgan Kaufmann Publishers, 1987.

[11] J. Berri, Y. Atif, and R. Benlamri. "Time-Dependent learning", Proceedings of the 4th IEEE International Conference on Advanced Learning Technologies (ICALT'04), 2004, pp. 816-818.

[12] Python programming language, available [Online]: www.python.org, accessed on 10 February 2010.

[13] K. Peters, "Learning On the Move: Mobile Technologies in Business and Education", Australian Flexible Learning Framework. 2005.

[14] Indywiki, available [Online]: indywiki.sourceforge.net/index.htm, accessed on 10 February 2010. 